\title{
A SYSTEMATIC STUDY OF THE LOW ENERGY EFFECTS OF HEAVY PARTICLES IN THE STANDARD MODEL
}

\author{
Eduardo V FLORES \\ Randall Laboratorv of Phvsics, University of Michigan Ann Arbor, MI 48109, USA*
}

Received 7 January 1988

(Revised 29 March 1989)

\begin{abstract}
In this approach, at any loop order, the low energy effects of a heavy Higgs boson and a heavy fermion can be summarized by an effective lagrangian To the one-loop order, an effective lagrangian for the bosomic sector of the theory is constructed One fermion mass $1 \mathrm{~s}$ light $(\mathrm{m})$ and the other is heavy $(M)$ At the one-loop level heavy fermion mass effects proportional to $M^{2}$ and $\ln (M / m)$ have been found It is shown here that in the presence of gauge fields the infinities $1 / \epsilon$ of the nonlinear $\sigma$-model do not fully reproduce the $\ln \left(M_{\mathrm{H}}\right)$ of the linear $\sigma$-model
\end{abstract}

\section{Introduction}

The study of heavy particles is an important subject in elementary partıcle physics and much effort is being put into the search for heavy elementary particles Particles such as the Higgs boson or the top quark are fundamental because the standard model cannot be established without the confirmed existence of these particles

The pattern for the masses of particles indicates that new partıcles are likely to be heavier than the particles already found It seems that the new discoveries in particle physics will come from the search for the heavy partıcles Heavy particles are predicted by different theories of elementary particles, and it would be good to be able to produce them in a laboratory However, there is a real limit on the avalable energy in a laboratory Because of the physical limitations on the production of heavy partıcles in a laboratory, it is necessary to search for new, low energy effects that confirm the existence of these particles This paper can be seen as an alternative low energy approach to the study of heavy particles Here, one explores the fact that some radiative corrections to physical processes, within the standard model, are heavy mass dependent and can be of importance

From the theoretical point of view, the study of heavy particles is quite interesting In the calculation of a diagram where at least one heavy particle is involved, there are big simplifications These are a motivatıng factor to attempt an ambitious calculation When the heavy particle is integrated out, there remains an effective lagrangian for each loop order This effective lagrangian can be found with

*New address Department of Physical Sciences, Glassboro State College, Glassboro, NJ 08028 USA 
relatively little effort, and from it any process involving heavy particles can be obtained

The effective lagrangian found here can be constructed at any loop order The one-loop calculation presented in this paper is meant to be an example of the technique explained below The $S$-matrix elements $(p=m)$ agree with the calculatıon of Appelquist and Bernard [2] at one-loop level, in the presence of a heavy Higgs boson

This paper will only consider a pair of quarks but, with minor changes, the results can be applied to leptons One of the two quark masses will be assumed to be much larger than the other mass and much larger than the external momentum $p$ The mass of the Higgs boson will also be considered to be much larger than the mass of the vector bosons and the external momentum $p$ The standard model consists of fermion, scalar boson and vector boson fields The lagrangian is

$$
\mathscr{L}=\mathscr{L}_{\text {fermions }}+\mathscr{L}_{\text {Higgs }}+\mathscr{L}_{\text {gauge bosons }}
$$

The part of the above lagrangian necessary for this work is

$$
\begin{aligned}
\mathscr{L}_{\text {quarks }}= & (\bar{t} \bar{b})_{\mathrm{L}} l \gamma_{\mathrm{L} \mu}^{\mu}\left(\begin{array}{l}
t \\
b
\end{array}\right)_{\mathrm{L}}+\bar{t}_{\mathrm{R}} l \gamma^{\mu} D_{\mathrm{R} \mu} t_{\mathrm{R}}+\bar{b}_{\mathrm{R}} l \gamma^{\mu} D_{\mathrm{R} \mu} b_{\mathrm{R}} \\
& -H\left[(\bar{t} \bar{b})_{\mathrm{L}}\left(\begin{array}{c}
\phi_{0} \\
\phi_{-}
\end{array}\right) t_{\mathrm{R}}+\mathrm{h} \mathrm{c}\right]-h\left[(\bar{t} \bar{b})_{\mathrm{L}}\left(\begin{array}{l}
\phi_{+} \\
\phi_{0}^{*}
\end{array}\right) b_{\mathrm{R}}+\mathrm{h} \mathrm{c}\right], \\
\mathscr{L}_{\mathrm{H} 1 \mathrm{ggs}}= & \left(D_{\mathrm{L}}^{\mu} \Phi\right)^{\dagger}\left({ }_{\mathrm{L} \mu} \Phi\right)-\frac{1}{8}\left(M_{\mathrm{H}}^{2} / f^{2}\right)\left(2 \Phi^{\dagger} \Phi-f^{2}\right)^{2}, \\
\mathscr{L}_{\text {gauge bosons }}= & -\frac{1}{4} F^{\mu \nu a} F_{\mu \nu}^{a}-\frac{1}{4} B^{\mu \nu} B_{\mu \nu},
\end{aligned}
$$

where $t$ and $b$ are the heavy and light fermions respectively $H$ and $h$ respectively are the large and small coupling constants for the interactions between quarks and scalar bosons The scalar bosons are described by a complex doublet,

$$
\Phi=\left(\begin{array}{c}
\phi_{0} \\
\phi_{-}
\end{array}\right),
$$

which has a self-coupling constant of $\frac{1}{8}\left(M_{\mathrm{H}}^{2} / f^{2}\right)$ Notice that $M_{\mathbf{H}}^{2}$ is the tree-level mass of the Higgs bosons

\section{The effective lagrangian}

The pattern of masses of fermion families seems to indicate the existence of a $t$ quark heavier than any other known quark At low energy, the heavier quark cannot be produced in a laboratory, but its presence may be felt through quantum effects These effects can be summarized by an effectıve lagrangıan To define the structures 
present in the effective lagrangian, one can use the path integral First, though, it is convenient to look at the equations of motion for $t_{\mathrm{L}}$ and $t_{\mathrm{R}}$,

$$
\left(\begin{array}{c}
t_{\mathrm{R}} \\
t_{\mathrm{L}}
\end{array}\right)=\frac{1}{p^{2}-M^{2}}\left(\begin{array}{ccc}
-\gamma p & M \\
M & -\gamma & p
\end{array}\right)\left(\begin{array}{c}
H\left[\phi_{+} b_{\mathrm{L}}+\left(\phi_{0}^{*}-f / \sqrt{2}\right) t_{\mathrm{L}}\right] \\
H\left(\phi_{0}-f / \sqrt{2}\right) t_{\mathrm{R}}-h \phi_{+} b_{\mathrm{R}}
\end{array}\right)+\left(\begin{array}{c}
t_{\mathrm{R}_{0}} \\
t_{\mathrm{L}_{0}}
\end{array}\right)
$$

The above equations have been obtained from $\mathscr{L}_{\text {quarks }}$ with no gauge fields Note that in this equation $M=(f / \sqrt{2}) H, f / \sqrt{2}=\left\langle\phi_{0}\right\rangle$, and $t_{\mathrm{L}_{0}}$ and $t_{\mathrm{R}_{0}}$ are the solutions of the homogeneous equations

$$
\left(\begin{array}{ccc}
\gamma & p & M \\
M & \gamma & p
\end{array}\right)\left(\begin{array}{c}
t_{\mathrm{R}_{0}} \\
t_{\mathrm{L}_{0}}
\end{array}\right)=0
$$

In the case where the $t$ quark is not produced because of its heavy mass $(M \gg p)$, the free fields $t_{\mathrm{L}_{0}}$ and $t_{\mathrm{R}_{0}}$ are set equal to zero and the above equations reduce to

$$
\left(\begin{array}{c}
t_{\mathrm{R}} \\
t_{\mathrm{L}}
\end{array}\right)=\left(\begin{array}{ll}
0 & 1 \\
1 & 0
\end{array}\right)\left(\begin{array}{c}
-(\sqrt{2} / f)\left[\phi_{+} b_{\mathrm{L}}+\left(\phi_{0}^{*}-f / \sqrt{2}\right) t_{\mathrm{L}}\right] \\
-(\sqrt{2} / f)\left(\phi_{0}-f / \sqrt{2}\right) t_{\mathrm{R}}
\end{array}\right)+\mathrm{O}(1 / H)
$$

or

$$
t_{\mathrm{R}}=0, \quad t_{\mathrm{L}}=-\left(\phi_{+} / \phi_{0}^{*}\right) b_{\mathrm{L}}
$$

The last equation can be rewritten as

$$
\left(\begin{array}{ll}
\phi_{0}^{*} & \phi_{+}
\end{array}\right)\left(\begin{array}{l}
t \\
b
\end{array}\right)_{\mathrm{L}}=0
$$

which is an $S U(2)_{L}$ invariant constraint [1] A similar analysis shows that the Higgs sector develops an $\mathrm{SU}(2)_{\mathrm{L}} \times \mathrm{SU}(2)_{\mathrm{R}}$ invariant constraint

$$
\sigma^{2}+\pi_{a}^{2}=f^{2}, \quad \text { or } \quad \Phi^{\dagger} \Phi=f^{2} / 2
$$

in the large Higgs mass limit [2]

It is necessary to expand the fermion and boson fields in the path integral about $t_{\mathrm{cl}}, b_{\mathrm{cl}}$ and $\Phi_{\mathrm{cl}}$, the solutions of the equations of motion In other words $t, b$ and $\phi$ in the basic lagrangian are replaced by

$$
t=t_{\mathrm{cl}}+\tilde{t}, \quad b=b_{\mathrm{cl}}+\tilde{b}, \quad \phi=\phi_{\mathrm{cl}}+\tilde{\phi},
$$

where the tilde indicates a quantum fluctuation For the case under consideration, $t_{\mathrm{cl}}$ obeys, to the order of $1 / M$, the constraints

$$
t_{\mathrm{clR}}=0
$$


and

$$
\left(\begin{array}{ll}
\phi_{0}^{*} & \phi_{+}
\end{array}\right)_{\mathrm{cl}}\left(\begin{array}{l}
t_{\mathrm{cl}} \\
b_{\mathrm{cl}}
\end{array}\right)_{\mathrm{L}}=0
$$

$\Phi_{\mathrm{cl}}$ obeys, to the order of $1 / M_{\mathrm{H}}^{2}$, the constraint

$$
\sigma_{\mathrm{cl}}^{2}+\pi_{\mathrm{cl}}^{2}=f^{2}
$$

The structures present in the effective lagrangian are written in terms of fields that obey the equations of motion If these fields are the exact solution of the equations of motion, then the effective lagrangian is expected to have the invariances of the original lagrangian Fields that are only an approximate solution to the equations of motion will give rise to effective lagrangians with non-invariant terms Thus the present problem is bound to have non-invariant terms in the effective lagrangian

In order to find the symmetries of the effective lagrangian in the large mass limit, one can expand the fields in the path integral about the above approximate solutions to the equations of motion The result of this expansion gives

$$
\begin{aligned}
\exp \left[\imath W\left(J, \eta, b_{\mathrm{cl}}, \phi_{\mathrm{cl}}\right)\right]= & \exp \left\{\int \mathrm{d}^{4} x \mathscr{L}_{\mathrm{Higgs}}+\mathscr{L}_{\text {quarks }}+J \phi+\eta b+\mathrm{O}\left(\frac{1}{M}, \frac{1}{M_{\mathrm{H}}^{2}}\right)\right\} \\
& \times \int \mathrm{d} \tilde{t} \mathrm{~d} \tilde{b} \mathrm{~d} \tilde{\phi} \exp \left\{l \int \mathrm{d}^{4} x \mathscr{L}^{\prime}+\mathrm{O}\left(\frac{1}{M}, \frac{1}{M_{\mathrm{H}}^{2}}\right)\right\},
\end{aligned}
$$

where $\mathscr{L}_{\text {quarks }}$ and $\mathscr{L}_{\text {Higgs }}$ are the original lagrangians after the constraints are replaced These two are relatively simple and they generate all the tree diagrams The loop diagrams are represented by $\mathscr{L}^{\prime}(t, \tilde{t}, b, \tilde{b}, \phi, \tilde{\phi})$, and it has pieces that are covariant and noncovariant with respect to the relevant transformations It can be shown $[1,3]$ that there are only two noncovariant structures in $\mathscr{L}^{\prime}$ These structures are $X$ and $\Sigma_{0}$ The structures $X$, due to the heavy $t$-fermion, is

$$
X=\frac{1}{\phi_{0}}\left(-\gamma^{\mu} \frac{1}{l} \partial_{\mu} t_{\mathrm{L}}+h \phi_{+} b_{\mathrm{R}}\right)
$$

$\Sigma_{0}$ is due to the heavy Higgs boson To understand the structure of $\Sigma_{0}$ consider the equation of motion for the $\sigma$-field

$$
\partial^{2} \sigma+\{\text { gauge fields, fermıon fields, etc }\}=-\left(M_{\mathrm{H}}^{2} / 2 f^{2}\right)\left(\sigma^{2}+\pi^{2}-f^{2}\right) \sigma,
$$

or

$$
\frac{1}{\sigma}\left[\partial^{2} \sigma+\{\text { gauge fields, fermion fields, etc }\}\right]=-\left(M_{\mathrm{H}}^{2} / 2 f^{2}\right)\left(\sigma^{2}+\pi^{2}-f^{2}\right)
$$

The left-hand side of the last equation is $\Sigma_{0}$ 
This paper claims that the structures in the effectıve lagrangian have the general form

$$
\alpha(\text { Invariant structures }) \times(X)^{n} \times\left(\Sigma_{0}\right)^{m}
$$

where the $\alpha$ 's are constants and $m$ and $n$ are positive integers or zero

However, under $\mathrm{SU}(2)_{\mathrm{L}}$ rotations, the variation of $X$ is proportional to the external currents in such a way that the variation of $X$ vanishes when the momentum is on-shell $\left(p^{2}=m^{2}\right)$ and the currents are zero [1] A similar result was found [2,3] for $\Sigma_{0}$ Therefore $\mathscr{L}^{\prime}$ is on-shell invariant Therefore, if one only needs on-shell Green functions one does not need to include $X$ or $\Sigma_{0}$

When the gauge fields are introduced, one needs to make sure that the explicit gauge symmetry of the effectıve lagrangian is preserved A gauge-fixıng term can give rise to non-ınvariant terms in the effective lagrangian A technique that preserves explicit gauge symmetries of the effective lagrangian exists and is called the background gauge field technique [4]

Another thing one needs to determine before writıng the structures of the effective lagrangian is the upper limit for the number of derivatives present in these structures The number of derivatives is determined by the momentum expansion of a Feynman diagram Standard power countıng techniques show that the maximum power $(n)$ for the external momentum expansion of a diagram, in the presence of a heavy particle, is given by

$$
n=2(L+1)-n_{\mathrm{f}},
$$

where $n_{\mathrm{f}}$ is the number of external fermion pairs and $L$ is the number of loops

\section{The fermion loop case}

One can classify one-loop processes into two categories The first category contains diagrams with no external fermions, and the second contains diagrams with external fermions This paper will consider only the first category Therefore the non-invariant structure $X$, which contains external fermions, will not appear in the effective lagrangian calculated here

There are two kinds of one-loop diagrams with no external fermions One kind contains fermion loops only and the other contains boson loops only This fact breaks the problem into two independent calculations In other words the fermion loop effects are summarized by one effective lagrangian and the boson loop effects are summarızed by another independent effectıve lagrangian

The path-integral shows that in the absence of external fermions the effective lagrangian is invariant under $\mathrm{SU}(2)_{\mathrm{L}}$ transformations The non-invariant structure $X$ is not present Only invariant structures need to be determined The upper limit ( $n$ ) for the number of derivatives in the one-loop effective lagrangian is four In the 
lımıt of large $\sigma$-mass, the $S U(2)_{L} \times U(1)$ invariant structures with two derivatives are

$$
\mathscr{L}_{1}^{\prime}=a_{1}\left(D_{\mu} \Phi\right)^{\dagger}\left(D^{\mu} \Phi\right), \quad \mathscr{L}_{2}^{\prime}=a_{2}\left[\left(D_{\mu} \Phi\right)^{\dagger} \Phi\right]\left[\Phi^{\dagger} D^{\mu} \Phi\right],
$$

where $D_{\mu}$ is the appropriate covariant derivative for $\Phi$, explicitly

$$
D_{\mu}=\partial_{\mu}-1 \frac{1}{2} g^{\prime} B_{\mu}+i \frac{1}{2} g \tau^{a} A_{\mu}^{a}
$$

In the limit of large $\sigma$-mass, the $\mathrm{SU}(2)_{\mathrm{L}} \times \mathrm{U}(1)$ invariant structures with four derivatives are

$$
\begin{aligned}
& \mathscr{L}_{1}=b_{1}\left(D_{\mu} D^{\mu} \Phi\right)^{\dagger}\left(D_{\nu} D^{\nu} \Phi\right) \\
& \mathscr{L}_{2}=b_{2}\left(\Phi^{\dagger} D_{\mu} D^{\mu} \Phi\right)\left[\left(D_{\nu} D^{\nu} \Phi\right)^{\dagger} \Phi\right] \\
& \mathscr{L}_{3}=b_{3}\left\{\left(\Phi^{\dagger} D_{\mu} \Phi\right)\left[\left(D^{\mu} D_{\nu} D^{\nu} \Phi\right)^{\dagger} \Phi\right]+\mathrm{h} \mathrm{c}\right\} \\
& \mathscr{L}_{4}=b_{4}\left\{\left(\Phi^{\dagger} D_{\mu} \Phi\right)\left[\left(D^{\nu} D^{\mu} \Phi\right)^{\dagger} D_{\nu} \Phi\right]+\mathrm{h} \mathrm{c}\right\} \\
& \mathscr{L}_{5}=b_{5}\left\{\left(\Phi^{\dagger} D_{\mu} \Phi\right)\left[\left(D_{\nu} D^{\nu} \Phi\right)^{\dagger} D^{\mu} \Phi\right]+\mathrm{h} \mathrm{c}\right\} \\
& \mathscr{L}_{6}=b_{6}\left\{\left(\Phi^{\dagger} D_{\mu} \Phi\right)\left[\left(D_{\nu} \Phi\right)^{\dagger} \Phi\right]\left[\left(D^{\mu} D^{\nu} \Phi\right)^{\dagger} \Phi\right]+\mathrm{h} \mathrm{c}\right\} \\
& \mathscr{L}_{7}=b_{7}\left\{\left(\Phi^{\dagger} D_{\mu} \Phi\right)\left[\left(D^{\mu} \Phi\right)^{\dagger} \Phi\right]\left[\left(D_{\nu} D^{\nu} \Phi\right)^{\dagger} \Phi+\Phi^{\dagger} D_{\nu} D^{\nu} \Phi\right]\right\} \\
& \mathscr{L}_{8}=b_{8}\left\{\left[\Phi^{\dagger} D_{\mu} \Phi\right]\left[\left(D^{\mu} \Phi\right)^{\dagger} \Phi\right]\right\}^{2} \\
& \mathscr{L}_{9}=b_{9}\left\{\left[\left(D_{\mu} \Phi\right)^{\dagger} D_{\nu} \Phi\right]\left(\Phi^{\dagger} D^{\mu} D^{\nu} \Phi\right)+\mathrm{h} \mathrm{c}\right\} \\
& \mathscr{L}_{10}=b_{10}\left\{\left(\Phi^{\dagger} D_{\mu} \Phi\right)\left[\left(D^{\mu} D^{\nu} \Phi\right)^{\dagger} D_{\nu} \Phi\right]+\mathrm{h} \mathrm{c}\right\} \\
& \mathscr{L}_{A}=b_{A} F_{\mu \nu}^{a} F^{\mu \nu a}, \\
& \mathscr{L}_{11}=b_{11}\left(\Phi^{\dagger} F_{\mu \nu} \Phi\right)\left[\left(F^{\mu \nu} \Phi\right)^{\dagger} \Phi\right] \\
& \mathscr{L}_{12}=b_{12}\left\{\left[\left(D_{\mu} \Phi\right)^{\dagger} D_{\nu} \Phi\right]\left(\Phi^{\dagger} F^{\mu \nu} \Phi\right)-\mathrm{h} \mathrm{c}\right\} \\
& \mathscr{L}_{B}=b_{B} B_{\mu \nu} B^{\mu \nu}, \\
& \mathscr{L}_{13}=b_{13} B_{\mu \nu}\left(\Phi^{\dagger} F^{\mu \nu} \Phi\right) \\
& \mathscr{L}_{14}=b_{14} B_{\mu \nu}\left[\left(D^{\mu} \Phi\right)^{\dagger} D_{\nu}^{\nu} \Phi-\mathrm{h} \mathrm{c}\right]
\end{aligned}
$$




\section{A SUMMARY OF THE CALCULATION}

To make the calculation simpler, one first sets the gauge-coupling constants $g$ and $g^{\prime}$ equal to zero so that the remaining lagrangian has only fermions and scalars In this limit there are two structures with two derivatives, $\mathscr{L}_{1}^{\prime}$ and $\mathscr{L}_{2}^{\prime}$, and there are ten structures with four derivatives, $\mathscr{L}_{1}, \mathscr{L}_{2}, \quad, \mathscr{L}_{10}$ The two structures with two derivatives are independent of each other In this case, independent means that a structure cannot be obtained from another by addıng surface integrals, the surface is at infinity where the integrand is assumed to be zero Out of the ten structures with four derivatives, only nine are independent because in this limit we have $\mathscr{L}_{4}=\mathscr{L}_{10}$ Furthermore, one only needs to choose eight out of the nine structures to reproduce any one-loop process [1] For this reason, the coefficient $b_{9}$ of structure $\mathscr{L}_{9}$ has been chosen to be zero The one-loop calculation needed to determine the coefficients of these structures has been summarized in table 1 The corresponding calculation from the effective lagrangian has been summarized in table $2 \mathrm{~A}$ comparison of the two tables results in the equations that determine the coefficients

When the gauge fields are "turned on", gauge invariance becomes local (a function of $x$ ) The derivatives in $\mathscr{L}_{1}^{\prime}, \mathscr{L}_{2}^{\prime}, \mathscr{L}_{1}, \mathscr{L}_{2}, \quad$ and $\mathscr{L}_{10}$ become covariant derivatives Covariant derivatives do not commute $\left(D_{\mu} D_{\nu} \neq D_{\nu} D_{\mu}\right)$, thus new structures appear There are seven new structures, $\mathscr{L}_{10}, \mathscr{L}_{11}, \mathscr{L}_{12}, \mathscr{L}_{13}, \mathscr{L}_{14}, \mathscr{L}_{A}$, and $\mathscr{L}_{B}$ The calculation that determines the new coefficients has been summarized in table 3, and the corresponding contribution from the structures of the effective lagrangian is summarized in table $4 \mathrm{~A}$ final comparison of the results written in the tables gives

$$
\begin{aligned}
& a_{1}=\frac{1}{(4 \pi)^{2}} \frac{1}{f^{2}} 2\left[\frac{1}{\epsilon}+\frac{1}{2}-\gamma-\ln \left(\pi M^{2} / \mu^{2}\right)\right]\left(M^{2}+m^{2}\right), \\
& a_{2}=\frac{1}{(4 \pi)^{2}} \frac{1}{f^{4}} 2\left[-\left(M^{2}+m^{2}\right)+2 m^{2} \ln \frac{M^{2}}{m^{2}}\right], \\
& b_{1}=\frac{1}{(4 \pi)^{2}} \frac{1}{f^{2}}\left(\frac{2}{3}\right), \quad b_{2}=\frac{1}{(4 \pi)^{2}} \frac{1}{f^{4}}\left(-\frac{2}{3}\right), \quad b_{3}=\frac{1}{(4 \pi)^{2}} \frac{1}{f^{4}}\left(-\frac{1}{3}\right), \\
& b_{4}=\frac{1}{(4 \pi)^{2}} \frac{1}{f^{4}}\left(-\frac{8}{9}+\frac{4}{3} \ln \frac{M^{2}}{m^{2}}\right), \quad b_{5}=\frac{1}{(4 \pi)^{2}} \frac{1}{f^{4}}\left(\frac{17}{9}-\frac{4}{3} \ln \frac{M^{2}}{m^{2}}\right), \\
& b_{6}=\frac{1}{(4 \pi)^{2}} \frac{1}{f^{6}}\left(-\frac{4}{3}\right), \quad b_{7}=\frac{1}{(4 \pi)^{2}} \frac{1}{f^{6}}(0), \quad b_{8}=\frac{1}{(4 \pi)^{2}} \frac{1}{f^{8}}(0), \\
& b_{9}=\frac{1}{(4 \pi)^{2}} \frac{1}{f^{4}}(0), \quad b_{10}=\frac{1}{(4 \pi)^{2}} \frac{1}{f^{4}}(-2), \quad
\end{aligned}
$$




$$
\begin{aligned}
& b_{11}=\frac{1}{(4 \pi)^{2}} \frac{1}{f^{4}} g^{2}\left(\frac{5}{18}-\frac{1}{6} \ln \frac{M^{2}}{m^{2}}\right), \quad b_{12}=\frac{1}{(4 \pi)^{2}} \frac{1}{f^{4}}(\mathrm{lg})\left(-\frac{1}{18}+\frac{1}{3} \ln \frac{M^{2}}{m^{2}}\right), \\
& b_{13}=\frac{1}{(4 \pi)^{2}} \frac{1}{f^{2}}\left(g g^{\prime}\right) \frac{1}{3}\left(-\frac{1}{2}+\frac{1}{6} \ln \frac{M^{2}}{m^{2}}\right), \quad b_{14}=\frac{1}{(4 \pi)^{2}} \frac{1}{f^{2}}\left(\mathrm{lg}^{\prime}\right) \frac{1}{18}\left(\frac{17}{2}+\ln \frac{M^{2}}{m^{2}}\right) \\
& b_{A}=\frac{1}{(4 \pi)^{2}} g^{2}\left(-\frac{1}{12}\right)\left[\frac{1}{\epsilon}+\frac{1}{3}-\gamma-\ln \left(\pi \frac{M^{2}}{\mu^{2}}\right)\right] \\
& b_{B}=\frac{1}{(4 \pi)^{2}} g^{\prime 2} \frac{1}{12}\left[-\frac{11}{9}\left(\frac{1}{\epsilon}-\gamma-\ln \left(\pi \frac{M^{2}}{\mu^{2}}\right)\right)+\frac{1}{2}-\frac{5}{18} \ln \frac{M^{2}}{m^{2}}\right]
\end{aligned}
$$

\section{PHYSICAL EFFECTS}

The large heavy fermion mass effects are of two types - one proportional to $M^{2}$ and the other proportional to $\ln (M / m)$ If the external momentum $p$ is smaller than the heavy mass $M$, then these results can be applied to the analysis of physical processes from a particle accelerator The results found here are not applicable in the limit where the two fermion masses $M$ and $m$ are equal

One can first consider the heavy mass $M^{2}$ effects There are two structures which contain these types of effects, one is $\mathscr{L}_{1}^{\prime}$ and the other is $\mathscr{L}_{2}^{\prime}$ The first structure $\mathscr{L}_{1}^{\prime}$ is of the form of the original lagrangian, and it can be removed by renormalization On the contrary, the second structure $\mathscr{L}_{2}^{\prime}$ is a new structure and cannot be removed by renormalization Thus, the processes present in $\mathscr{L}_{2}^{\prime}$ can have physical significance Vertices with three external legs that come from the physically relevant structure $\mathscr{L}_{2}^{\prime}$ are

$$
\begin{array}{r}
-\frac{1}{(4 \pi)^{2}} \frac{M^{2}}{f^{2}}\left[l \frac{g}{2 \cos \theta} Z_{\mu}\left(\phi^{+} \partial^{\mu} \phi^{-}-\mathrm{c} \mathrm{c}\right)-g \partial_{\mu} \pi_{3}\left(W_{-}^{\mu} \phi^{+}+\mathrm{c} \mathrm{c}\right)\right. \\
\left.+\frac{g^{2}}{2 \cos \theta} f Z_{\mu}\left(W_{-}^{\mu} \phi^{+}+\mathrm{c} \mathrm{c}\right)\right]
\end{array}
$$

This one loop correction has a factor of $\left(1 /(4 \pi)^{2}\right) M^{2} / f^{2}$ Therefore, only a large value for $M$ of the order of $100 \mathrm{GeV}$ or higher will be of importance

At low energies the $\mathrm{ZW}^{-} \phi^{+}$-vertex can be read from $\mathscr{L}_{2}^{\prime}$ and the result is

$$
-\frac{1}{(4 \pi)^{2}} \frac{M^{2}}{f^{2}} g M_{Z} g_{\mu \nu}
$$

This can be a particularly big value because it is independent of the external low 
TABLE 1

One-fermion-loop calculation of various Green functions in the limut $g=g^{\prime}=0$ (all momenta flow into vertices)

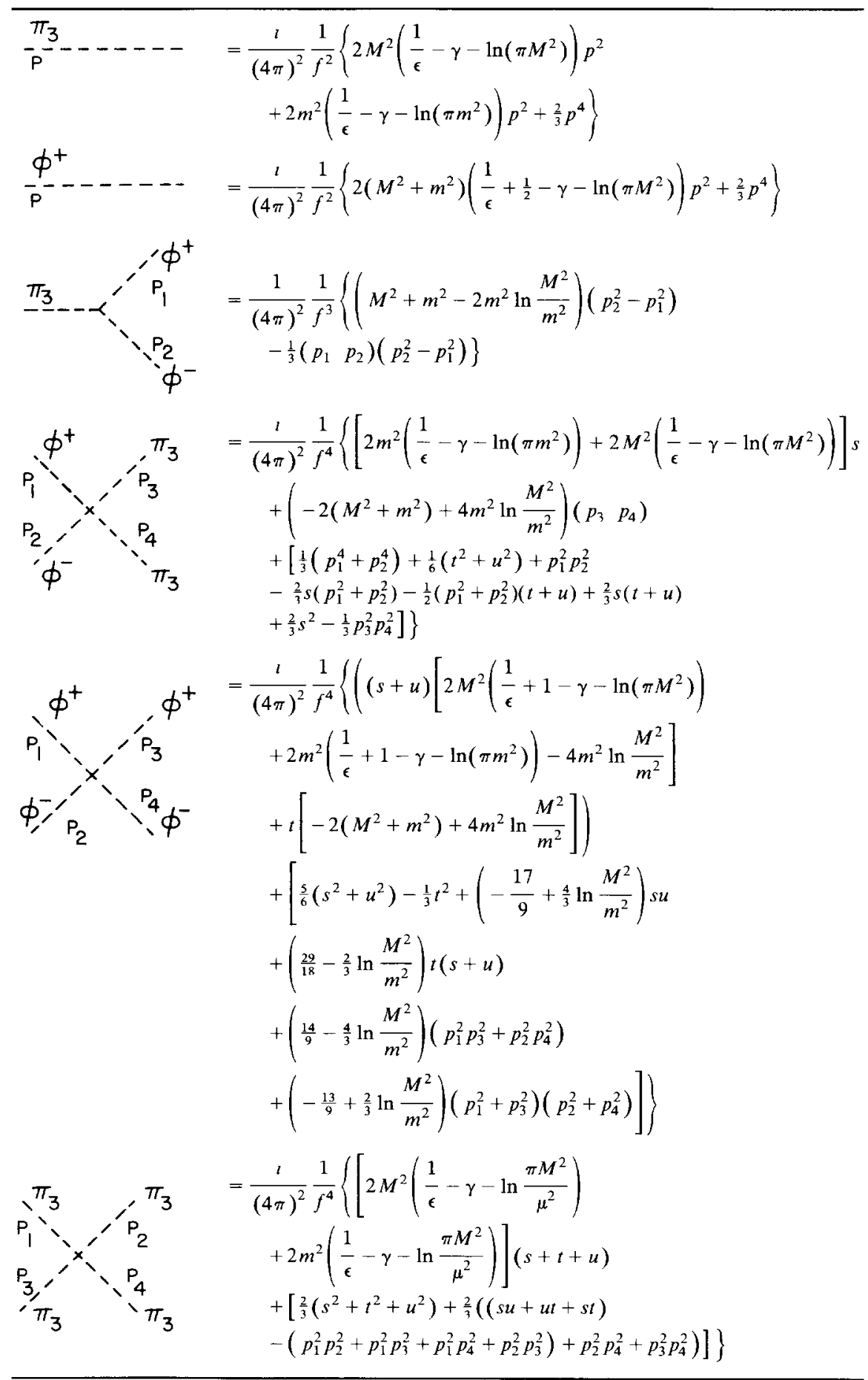


294

EV Flores / Heavy particles

TABLE 2

Contribution of structures $\mathscr{L}_{1}^{\prime}, \quad, \mathscr{L}_{9}$ to various Green functions (all momenta flow into vertices) (calculated in the $g=0$ and $g^{\prime}=0 l_{\mathrm{lmit}}$ )
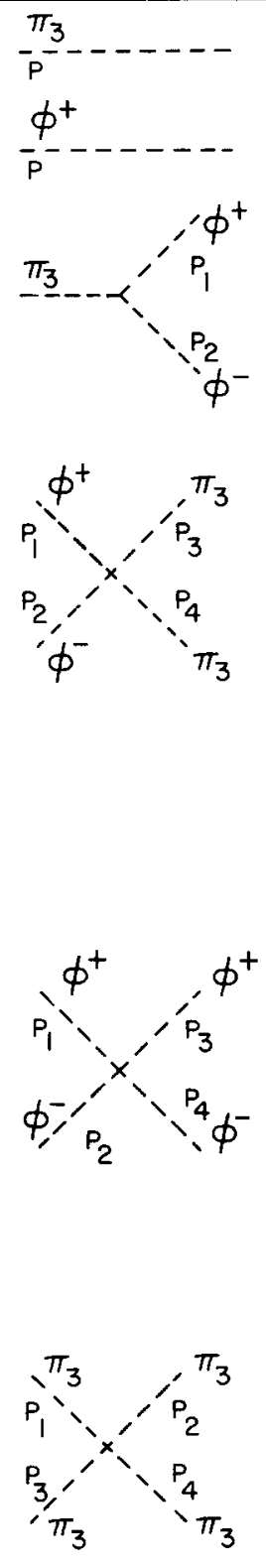

$=\imath b_{1} p^{4}+\imath\left(a_{1}-\frac{1}{2} a_{2} f^{2}\right) p^{2}$

$=\imath b_{1} p^{4}+\imath a_{1} p^{2}$

$$
\begin{aligned}
=- & f\left(\left(\frac{1}{2} b_{2}-b_{3}\right)\left(p_{2}^{4}-p_{1}^{4}\right)\right. \\
& +\left(b_{2}-\frac{3}{2} b_{3}-\frac{1}{2} b_{4}-\frac{1}{2} b_{5}\right) p_{1} p_{2}\left(p_{2}^{2}-p_{1}^{2}\right) \\
& +f\left\{\frac{1}{2} a_{2}\left(p_{2}^{2}-p_{1}^{2}\right)\right\}
\end{aligned}
$$

$=\imath\left\{\left[\frac{1}{2}\left(b_{2}-2 b_{3}\right)+b_{1}\right] s^{2}+\left(\frac{1}{2} b_{3}-\frac{1}{2} b_{4}-\frac{1}{2} b_{5}+\frac{1}{4} f^{2} b_{6}\right) u t\right.$ $+\left(\frac{1}{4} b_{3}-\frac{1}{4} b_{4}-\frac{1}{4} b_{5}\right)\left(t^{2}+u^{2}\right)$

$+\left(-\frac{1}{4} b_{3}-\frac{3}{4} b_{4}-\frac{3}{4} b_{5}+\frac{1}{8} f^{2} b_{6}+\frac{1}{4} f^{2} b_{7}\right) s(t+u)$

$-\left(-\frac{1}{4} b_{3}-\frac{3}{4} b_{4}-\frac{3}{4} b_{5}+\frac{1}{8} b_{6}+\frac{1}{4} b_{7}\right) s\left(p_{1}^{2}+p_{2}^{2}\right)$

$+\left(\frac{1}{2} b_{2}-\frac{1}{2} b_{3}+\frac{1}{2} b_{4}+\frac{1}{2} b_{5}-\frac{1}{8} f^{2} b_{6}-\frac{1}{4} f^{2} b_{7}\right)(t+u)\left(p_{1}^{2}+p_{2}^{2}\right)$

$+\left(-\frac{1}{2} b_{2}+\frac{1}{4} b_{3}-\frac{1}{4} b_{4}-\frac{1}{4} b_{5}+\frac{1}{8} f^{2} b_{6}+\frac{1}{4} f^{2} b_{7}\right)\left(p_{1}^{4}+p_{2}^{4}\right)$

$+\left(-b_{2}+\frac{1}{2} b_{3}-\frac{1}{2} b_{4}-\frac{1}{2} b_{5}+\frac{1}{2} f^{2} b_{7}\right) p_{1}^{2} p_{2}^{2}$

$\left.+\left(-b_{2}+b_{3}+b_{4}+b_{5}-\frac{1}{4} f^{2} b_{6}\right) p_{3}^{2} p_{4}^{2}\right\}$

$+\imath\left\{\left(\frac{a_{1}}{f^{2}}+\frac{1}{2} a_{2}\right) s+a_{2} p_{3} p_{4}\right\}$

$=l\left\{\left[\frac{1}{4}\left(b_{3}-b_{4}-b_{5}\right)+\frac{b_{1}}{f^{2}}\right]\left(s^{2}+u^{2}\right)\right.$

$-\frac{1}{2}\left(b_{3}-b_{4}-b_{5}\right) t^{2}$

$+\left(-b_{2}+\frac{1}{2} b_{3}+\frac{1}{2} b_{4}-\frac{1}{2} b_{5}\right) s u$

$+\left(b_{3}+b_{5}\right)\left(p_{1}^{2} p_{3}^{2}+p_{2}^{2} p_{4}^{2}\right)$

$+\left(-\frac{1}{2} b_{2}-\frac{1}{4} b_{3}-\frac{1}{4} b_{4}+\frac{1}{4} b_{5}\right) t(s+u)$

$\left.+\left(b_{2}-\frac{1}{2} b_{3}-\frac{1}{2} b_{5}\right)\left(p_{1}^{2}+p_{3}^{2}\right)\left(p_{2}^{2}+p_{4}^{2}\right)\right\}$

$+1\left\{\left(\frac{a_{1}}{f^{2}}-\frac{1}{2} a_{2}\right)(s+u)+a_{2} t\right\}$

$=l\left\{\left(\frac{1}{2} b_{2}-b_{3}+\frac{b_{1}}{f_{2}}\right)\left(s^{2}+t^{2}+u^{2}\right)\right.$

$+2\left(-b_{4}-b_{5}+\frac{1}{2} f^{2} b_{6}-\frac{1}{8} f^{4} b_{8}\right)[(s t+s u+u t)$

$\left.\left.-\left(p_{1}^{2} p_{2}^{2}+p_{1}^{2} p_{3}^{2}+p_{1}^{2} p_{4}^{2}+p_{2}^{2} p_{3}^{2}+p_{2}^{2} p_{4}^{2}+p_{3}^{2} p_{4}^{2}\right)\right]\right\}$

$+1\left\{\left(\frac{a_{1}}{f^{2}}+\frac{1}{2} a_{2}\right)(s+t+u)\right\}$ 
TABLE 3

One-termıon-loop calculation of various Green functions

(all momenta flow into vertices) (the first three Green functions are calculated in $g^{\prime}=0$ )

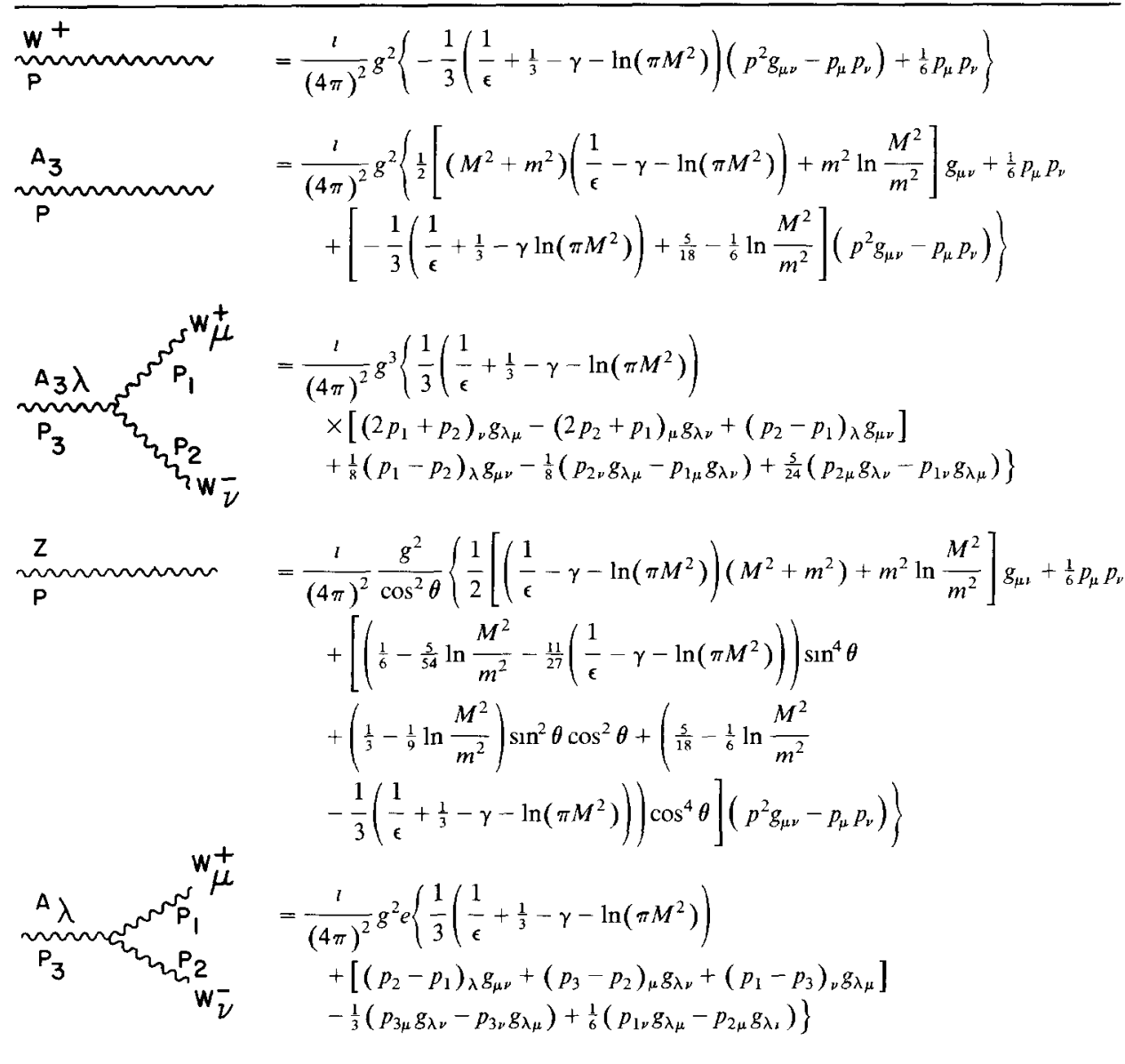

momentum, proportional to the mass of the Z-boson, and proportional to the mass squared of the heavy t-fermion $\mathrm{e}^{+} \mathrm{e}^{-}$machines should be able to provide data for this type of interaction The electron and the positron can produce the vector bosons $\mathrm{Z}$ and $\mathrm{W}$, and the scalar boson $\phi$ will decay into heavy fermions such as $b \bar{b}$, to which it couples strongly

The vertex $\mathrm{Z}^{+} \phi^{-}$can be produced in an electron-positron annihilation The value for this vertex can be read from $\mathscr{L}_{2}^{\prime}$ with a result equal to

$$
\frac{1}{(4 \pi)^{2}} \frac{M^{2}}{f^{2}} \frac{g}{2 \cos \theta}\left(p^{-}-p^{+}\right)_{\mu}
$$

The two scalar bosons will also decay into heavy fermıons 
TABLE 4

Contribution of the structures $\mathscr{L}_{10}, \quad, \mathscr{L}_{14}$ to various Green functions (all momenta flow into vertices) (the first three Green functions are calculated in $g^{\prime}=0$ )

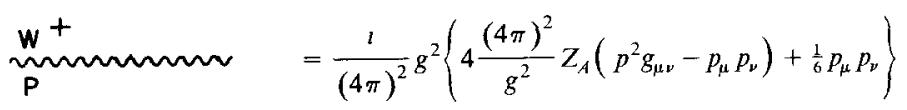

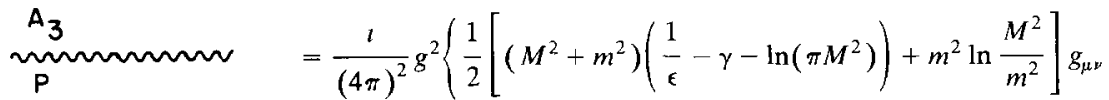
$\left.+\frac{1}{6} p_{\mu} p_{\nu}+\frac{(4 \pi)^{2}}{g^{2}}\left[Z_{A}+f^{4} b_{11}\right]\left(p^{2} g_{\mu \nu}-p_{\mu} p_{\nu}\right)\right\}$

${ }_{5} W_{\mu}^{+}=\frac{1}{(4 \pi)^{2}} g^{3}\left\{-4 \frac{(4 \pi)^{2}}{g^{2}} Z_{A}\left[\left(2 p_{1}+p_{2}\right)_{\nu} g_{\lambda \mu}-\left(2 p_{2}+p_{1}\right)_{\mu} g_{\lambda \nu}\right.\right.$

$\left.A_{3} \lambda 5^{5} \mathrm{P}_{\mathrm{P}_{1}}+\left(p_{2}-p_{1}\right)_{\lambda} g_{\mu \nu}\right]$

$\mathrm{P}_{3} \sum_{2} \mathrm{P}_{2} \quad-\frac{1}{16}(4 \pi)^{2} f^{4} b_{10}\left(p_{1}-p_{2}\right)_{\lambda} g_{\mu v}$

$+\left[\frac{1}{48}+\frac{1}{16}\left(\frac{17}{9}-\frac{4}{3} \ln \frac{M^{2}}{m^{2}}\right)\right.$

$\left.+(4 \pi)^{2}\left(-f^{4} b_{11}+\frac{1}{4} l f^{4} \frac{1}{g} b_{12}\right)\right]\left(p_{2 \nu} g_{\lambda \mu}-p_{1 \mu} g_{\lambda \nu}\right)$

$+\left[\frac{1}{16}\left(-\frac{8}{9}+\frac{4}{3} \ln \frac{M^{2}}{m^{2}}\right)\right.$

$\left.\left.+(4 \pi)^{2}\left(f^{4} \frac{1}{g^{2}} b_{11}-\frac{1}{4} l f^{4} \frac{1}{g} b_{12}\right)\right]\left(p_{2 \mu} g_{\lambda_{\nu}}-p_{1 \nu} g_{\lambda_{\mu}}\right)\right\}$

Z

บ

$$
\begin{aligned}
= & \frac{t}{(4 \pi)^{2}} \frac{g^{2}}{\cos ^{2} \theta}\left\{\frac{1}{2}\left[\left(\frac{1}{\epsilon}-\gamma-\ln \left(\pi M^{2}\right)\right)\left(M^{2}+m^{2}\right)+m^{2} \ln \frac{M^{2}}{m^{2}}\right] g_{\mu l}\right. \\
& +\frac{1}{6} p_{\mu} p_{\nu}+(4 \pi)^{2}\left[\left(4 \frac{1}{g^{2}} Z_{B}\right) \sin ^{4} \theta+\left(-2 f^{2} \frac{1}{g^{2}} b_{13}\right) \sin ^{2} \theta \cos ^{2} \theta\right. \\
& \left.\left.+\left(4 \frac{1}{g^{2}} Z_{A}+f^{4} \frac{1}{g^{2}} b_{11}\right) \cos ^{4} \theta\right]\left(p^{2} g_{\mu \nu}-p_{\mu} p_{\nu}\right)\right\}
\end{aligned}
$$

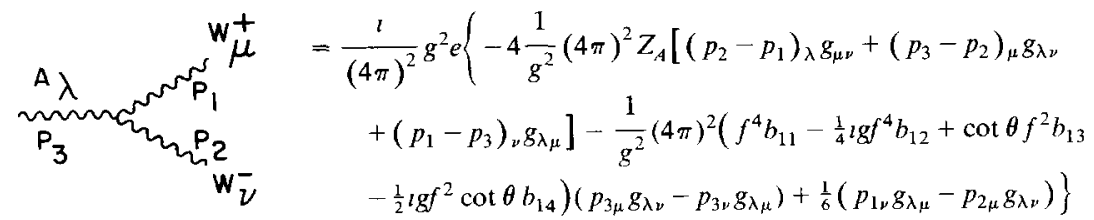


There are a number of processes in $\mathscr{L}_{2}^{\prime}$ with four and higher external legs The vertices with four external legs are

$$
\begin{aligned}
& -\frac{1}{(4 \pi)^{2}} \frac{M^{2}}{f^{2}} \frac{1}{f^{2}}\left\{\left[\frac{1}{2}\left(\pi_{3} \partial_{\mu} \pi_{3}\right)^{2}+\frac{1}{2}\left[\partial_{\mu}\left(\phi^{+} \phi^{-}\right)\right]^{2}+\pi_{3} \partial_{\mu} \pi_{3} \partial_{\mu}\left(\phi^{+} \phi^{-}\right)-\phi^{+} \phi^{-}\left(\partial_{\mu} \pi_{3}\right)^{2}\right]\right. \\
& \quad+\left[-M_{\mathrm{Z}} Z_{\mu}\left[\frac{1}{2} \pi_{3}^{2} \partial_{\mu} \pi_{3}-\phi^{+} \phi^{-} \partial_{\mu} \pi_{3}+\pi_{3} \partial_{\mu}\left(\phi^{+} \phi^{-}\right)\right]\right. \\
& \quad+\imath 2 M_{\mathrm{W}} \pi_{3} \partial_{\mu} \pi_{3}\left(W_{\mu}^{-} \pi+-\mathrm{cc}\right)+4 M_{\mathrm{W}} \partial_{\mu} \pi_{3} \phi^{+} \phi^{-}\left(\left(1+\sin ^{2} \theta / \cos \theta\right) Z_{\mu}+\sin \theta A_{\mu}\right) \\
& \left.\quad+\imath 2 M_{\mathrm{W}}\left(\phi^{+} \partial_{\mu} \phi^{-}-\mathrm{c} \mathrm{c}\right)\left(W^{-} \phi^{+}-\mathrm{c} \mathrm{c}\right)\right]+\left[2 M_{\mathrm{W}}^{2}\left(W^{-} \phi^{+}+\mathrm{cc}\right)^{2}-4 M_{\mathrm{W}}^{2} Z_{\mu} \phi^{+} \phi^{-}\right. \\
& \left.\left.\quad \times\left(\left(1+\sin ^{2} \theta\right) / \cos ^{2} \theta+\tan \theta A_{\mu}\right)-2 \imath\left(M_{\mathrm{W}}^{2} / \cos \theta\right) Z_{\mu} \pi_{3}\left(W^{-} \phi^{+}-\mathrm{c} \mathrm{c}\right)\right]\right\}
\end{aligned}
$$

The heavy mass $\ln (M / m)$ effects are more numerous than the $M^{2}$ effects The outstanding logarithmic effects are contained in the structures with four derivatives It is interesting to note that processes with less than four external legs do not have any heavy-mass $p^{4} \ln (M / m)$ corrections The first process with these type of corrections is the $4 \mathrm{~W}$ vertex, and from the effective lagrangian one reads the result for this interaction

$$
\begin{aligned}
\frac{1}{(4 \pi)^{2}} g^{4}\{[ & \left.\frac{1}{6}\left(\frac{1}{\epsilon}-\gamma-\ln \pi M^{2}\right)-\frac{1}{12}+\frac{1}{12} \ln \left(M^{2} / m^{2}\right)\right] \\
& \left.\times\left(2 g_{\alpha \beta} g_{\lambda \rho}-g_{\alpha \lambda} g_{\beta \rho}-g_{\alpha \rho} g_{\beta \lambda}\right)+\frac{1}{12}\left(g_{\alpha \lambda} g_{\beta \rho}+g_{\alpha \rho} g_{\beta \lambda}\right)\right\}
\end{aligned}
$$

The term proportional to the $\ln \left(M^{2} / \mathrm{m}^{2}\right)$ comes from the structure $\mathscr{L}_{11}$ which 1 not of the form of the original lagrangian, thus it has physical significance The larger the mass difference of a fermion doublet, the larger this effect becomes The $4 \mathrm{~W}$ vertex extracted here can be coupled to external light fermions and can mimic the $4 \mathrm{~W}$ vertex that comes from the original lagrangian This effect is probably the largest one-loop contribution to the $4 \mathrm{~W}$ vertex coming from fermions

There is also a vertex with four charged scalar particles The momentum structure and the heavy mass dependence can be found in table 1 The coupling constant between scalars and fermions is proportional to the mass of the fermions, thus heavy fermions will be the predominant decay mode for these scalars Leptonhadron and hadron-hadron colliders are better qualified to produce scalar bosons than $\mathrm{e}^{+} \mathrm{e}^{-}$colliders Processes with more than four external boson legs will be harder to observe experımentally, in any case these processes can be read from the effective lagrangian 


\section{The boson loop case}

Appelquist and Bernard [2] showed that an effective lagrangian bult out of $\mathrm{SU}(2)_{\mathrm{L}}$ gauge-invariant structures alone can only reproduce the $S$-matrix elements (with momentum restricted to $p^{2}=m^{2}$ ) of the SU(2) $L_{L}$ gauged $\sigma$-model in the large o-mass $\left(M_{\mathrm{H}}\right)$ limit On the other hand, Akhoury and Yao [3] found an effective lagrangian which reproduces not only the $S$-matrix elements but also the Green functions (with arbitrary external momentum) of the $\sigma$-model In their paper, Akhoury and Yao used path-integral methods to show that there is an extra non-invariant structure $\Sigma_{0}$ This structure arises in the limit of large $\sigma$-mass Invariant structures, together with $\Sigma_{0}$, are capable of reproducing all the Green functions of the theory In this paper, the techniques of Akhoury and Yao are applied to the study of the $\mathrm{SU}(2)_{\mathrm{L}} \times \mathrm{U}(1)$ gauged $\sigma$-model in the large $\sigma$-mass limit One finds that even in the presence of gauge fields there is only one non-invariant structure, which is the approprate generalization of $\Sigma_{0}$

The $S$-matrix elements obtained here agree with the $S$-matrix elements calculated by previous researchers in the subject $[2,5]$ It will be shown that there are small but fundamental differences between this calculation and the calculations by Longhitano [5], and Appelquist and Bernard [2] These differences are the following (1) some extra structure in the effective lagrangian, (11) the model in which calculations are performed, and (111) the freedom allowed to the external momentum

In the large $\sigma$-mass limit $\left(M_{\mathrm{H}} \rightarrow \infty\right)$, the linear $\sigma$-model becomes what is known as the nonlinear $\sigma$-model The nonlinear $\sigma$-model contains graphs with infinities that cannot be disentangled from the physical results When no gauge fields are present, the infinities can be shown [3] to represent some of the $\ln \left(M_{\mathrm{H}}\right)$ of the linear $\sigma$-model It will be shown that at the one-loop level in the presence of gauge fields these infinities do not fully reproduce the $\ln \left(M_{\mathrm{H}} / M_{\mathrm{W}}\right)$ of the linear model

Many experiments involve the measurement of cross sections of particles with mass and momentum smaller than the mass of vector bosons In these low energy experiments, vector bosons will not be the final states and they will contribute as virtual particles only Therefore, this paper considers a $S U(2)_{L} \times U(1)$ effective lagrangian valid not only at momentum $p^{2}=M_{\mathrm{W}}^{2}$ but also at small momentum

The technıques mentioned above are extremely helpful in calculatıng and justifying the construction of effective lagrangians, furthermore, these techniques are applicable beyond one loop

Power countıng shows that the one-boson-loop effectıve lagrangian can have structures with up to four derivatives Longhitano [5] wrote down all the possible $\mathrm{SU}(2)_{\mathrm{L}} \times \mathrm{U}(1)$ invariant structures of the non-linear $\sigma$-model with up to four derivatives His notation is kept here To specify the notation, define

$$
U=M / f=\sigma / f+\imath \tau^{a} \pi^{a} / f, \quad V_{\mu}=\left(D_{\mu} U\right) U^{\dagger}=-U\left(D_{\mu} U\right)^{\dagger}, \quad T=U \tau^{3} U^{\dagger},
$$


where $\tau^{a}$ are the Paul matrices and

$$
D_{\mu} U=\partial_{\mu} U+\imath g \frac{1}{2} \tau^{a} A_{\mu}^{a} U-\imath g^{\prime} \frac{1}{2} B_{\mu} U \tau^{3}
$$

is the covariant derivative of the unitless matrix $U$ Finally, the covariant derivative for $V_{\mu}$ is

$$
\mathscr{D}_{\mu} V_{\nu}=\partial_{\mu} V_{\nu}+\imath g\left[A_{\mu}, V_{\nu}\right]
$$

where $A_{\mu}=\frac{1}{2} \tau^{a} A_{\mu}^{a}$

The structures of dimension two are

$$
\mathscr{L}_{b 1}^{\prime}=\frac{1}{4} g^{2} f^{2} \beta_{1}\left[\operatorname{Tr}\left(V_{\mu} T\right)\right]^{2}, \quad \mathscr{L}_{b 2}^{\prime}=-\frac{1}{4} f^{2} \beta_{2} \operatorname{Tr}\left(V_{\mu} V^{\mu}\right), \quad \mathscr{L}_{b 3}^{\prime}=\beta_{3} \Sigma_{0}
$$

where $\Sigma_{0}$, the non-ınvariant structure, is

$$
\begin{aligned}
\Sigma_{0}=\left\{\frac { 1 } { \sigma _ { 0 } } \left[\partial^{2} \sigma_{0}-\right.\right. & g\left(A_{\mu}^{a} \partial^{\mu} \pi^{a}+\frac{1}{2} \partial_{\mu} A^{\mu a} \pi^{a}\right)+g^{\prime}\left(B_{\mu} \partial^{\mu} \pi^{3}+\frac{1}{2} \partial_{\mu} B^{\mu} \pi^{3}\right) \\
& \left.\left.+\frac{1}{2} g g^{\prime} \epsilon^{3 a b} \pi^{a} A_{\mu}^{b} B^{\mu}\right]+\frac{1}{2} g g^{\prime} A_{\mu}^{3} B^{\mu}-\frac{1}{4} g^{2} A_{\mu}^{a 2}-\frac{1}{4} g^{\prime 2} B_{\mu}^{2}\right\}
\end{aligned}
$$

The $C P$-invariant structures of dimension four are

$$
\begin{aligned}
& \mathscr{L}_{b 1}=\frac{1}{2} g^{2} \alpha_{1} B_{\mu \nu} \operatorname{Tr}\left(T F^{\mu \nu}\right), \quad \mathscr{L}_{b 2}=\frac{1}{2} l g \alpha_{2} B_{\mu \nu} \operatorname{Tr}\left(T\left[V^{\mu}, V^{\nu}\right]\right), \\
& \mathscr{L}_{b 3}=\lg \alpha_{3} \operatorname{Tr}\left(F_{\mu \nu}\left[V^{\mu}, V^{\nu}\right]\right), \quad \mathscr{L}_{b 4}=\alpha_{4}\left[\operatorname{Tr}\left(V_{\mu} V_{\nu}\right)\right]^{2}, \quad \mathscr{L}_{b 5}=\alpha_{5}\left[\operatorname{Tr}\left(V_{\mu} V^{\mu}\right)\right]^{2}, \\
& \mathscr{L}_{b 6}=\alpha_{6} \sin \theta_{\mathrm{W}} \operatorname{Tr}\left(V_{\mu} V_{\nu}\right) \operatorname{Tr}\left(T V^{\mu}\right) \operatorname{Tr}\left(T V^{\nu}\right), \quad \mathscr{L}_{b 7}=\alpha_{7} \sin \theta_{\mathrm{W}} \operatorname{Tr}\left(V_{\mu} V^{\mu}\right)\left[\operatorname{Tr}\left(T V_{\nu}\right)\right]^{2}: \\
& \mathscr{L}_{b 8}=\frac{1}{4} g^{2} \alpha_{8} \sin \theta_{\mathrm{W}}\left[\operatorname{Tr}\left(T F_{\mu \nu}\right)\right]^{2}, \quad \mathscr{L}_{b 9}=\frac{1}{2} l g \alpha_{9} \sin \theta_{\mathrm{W}} \operatorname{Tr}\left(T F_{\mu \nu}\right) \operatorname{Tr}\left(T\left[V^{\mu}, V^{\nu}\right]\right), \\
& \mathscr{L}_{b 10}=\frac{1}{2} \alpha_{10} \sin \theta_{\mathrm{W}}\left[\operatorname{Tr}\left(T V_{\mu}\right) \operatorname{Tr}\left(T V_{\nu}\right)\right]^{2}, \quad \mathscr{L}_{b 11}=\alpha_{11} \operatorname{Tr}\left[\left(\mathscr{D}_{\mu} V^{\nu}\right)^{2}\right], \\
& \mathscr{L}_{b 12}=\frac{1}{2} \alpha_{12} \sin \theta_{\mathrm{W}} \operatorname{Tr}\left(T \mathscr{D}_{\mu} \mathscr{D}_{\nu} V^{\nu}\right) \operatorname{Tr}\left(T V^{\mu}\right), \quad \mathscr{L}_{b 13}=\frac{1}{2} \alpha_{13} \sin \theta_{\mathrm{W}}\left[\operatorname{Tr}\left(T \mathscr{D}_{\mu} V_{\nu}\right)\right]^{2}, \\
& \mathscr{L}_{b 19}=\alpha_{19} \sin \theta_{\mathrm{W}} \Sigma_{0}\left[\operatorname{Tr}\left(T V_{\mu}\right)\right]^{2}, \quad \mathscr{L}_{b 20}=\alpha_{20} \Sigma_{0} \operatorname{Tr}\left(V_{\mu} V^{\mu}\right), \quad \mathscr{L}_{b 21}=\alpha_{21}\left(\Sigma_{0}\right)^{2}, \\
& \mathscr{L}_{b A}=-\frac{1}{2} Z_{A} \operatorname{Tr}\left(F_{\mu \nu} F^{\mu \nu}\right), \quad \mathscr{L}_{b B}=-\frac{1}{4} Z_{B} B_{\mu \nu} B^{\mu \nu}
\end{aligned}
$$




\section{A SUMMARY OF THE CALCULATION}

If one is interested only in the heavy-mass effects, then one can drop all the terms independent of the heavy mass $M_{\mathrm{H}}$ The structures $\mathscr{L}_{b 11}, \mathscr{L}_{b 12}$, and $\mathscr{L}_{b 13}$ contribute independently to the $p^{4}$ momentum dependence of two- and three-point functions of pure scalar graphs Graphs with two or three external scalar legs do not have momentum dependence of the form $p^{4} \ln \left(M_{\mathrm{H}}\right)$, thus $\mathscr{L}_{b 11}, \mathscr{L}_{b 12}$, and $\mathscr{L}_{b 13}$ should not be present

In the background gauge field method, one is free to choose any gauge without losing explicit gauge invariance This calculation has been performed in the $\alpha=0$

One-boson-loop calculations of various Green functions (all momenta flow into vertices)

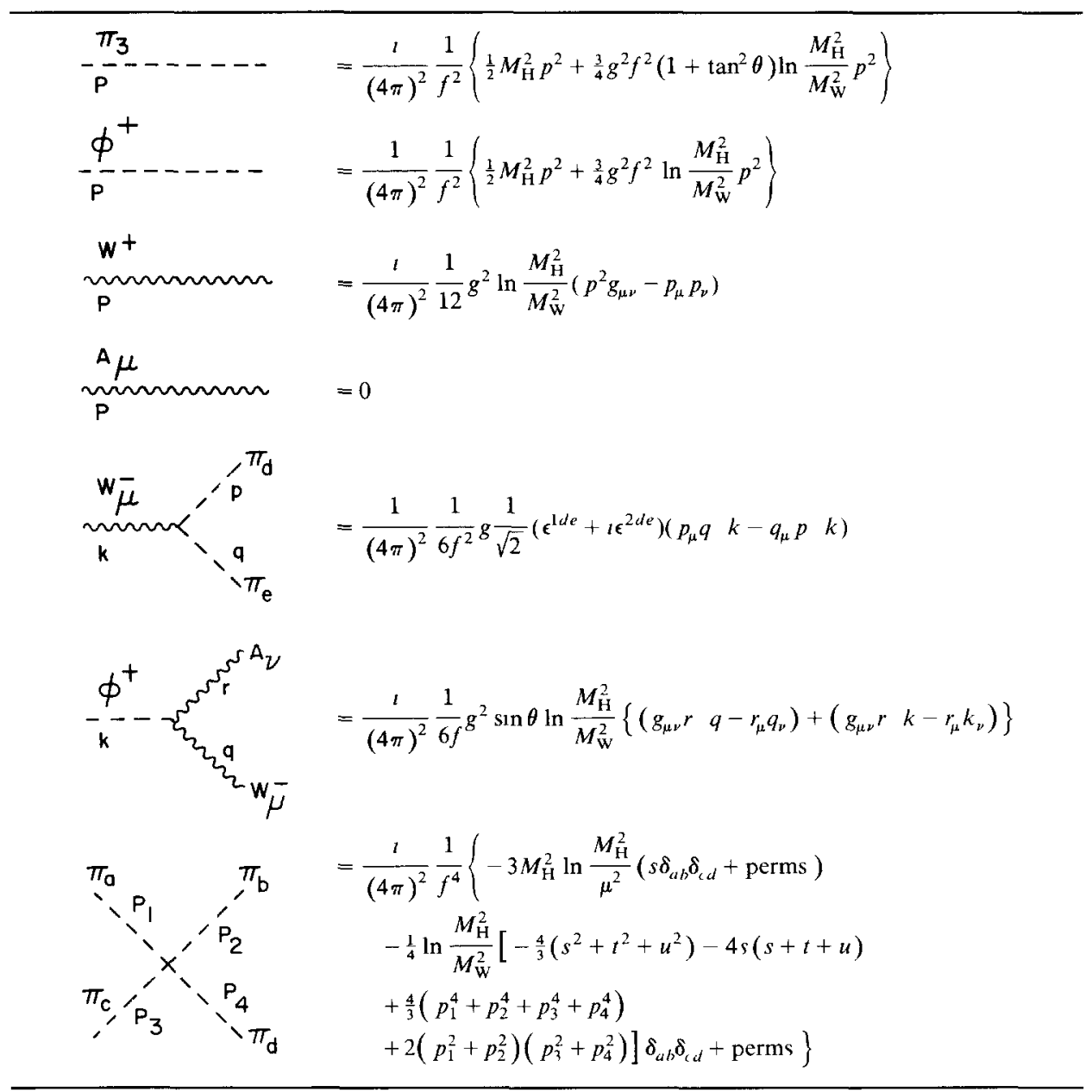


background gauge [4] In this gauge, one first needs to cancel every coefficient proportional to $1 / \alpha$ before settıng $\alpha$ equal to 0

The one-loop calculation, which determines $\beta_{1}$ through $Z_{B}$, resembles a calculation performed in the standard $\mathrm{R}_{\xi}=\infty$ gauge (Landau gauge) This fact justifies the on-shell one-loop computation by previous authors $[2,5]$, who calculated in the standard Landau gauge and assumed the existence of gauge-invariant structures for the effective lagrangian However, their assumption does not work beyond one loop The one-loop calculation of the coefficients of the structures of the effective lagrangian can be done by choosing graphs with internal scalar particles only, thus

TABLE 6

Contribution of the structures $\mathscr{L}_{b 1}^{\prime}, \quad, \mathscr{L}_{b \mathrm{~B}}$ to various Green functions (all momenta flow into vertices)

$$
\begin{aligned}
& -\pi_{3}----=l\left(\beta_{2}-2 g^{2} \beta_{1}\right) p^{2} \\
& \frac{\phi^{+}}{P} \frac{P^{-}}{P} \beta_{2} p^{2}
\end{aligned}
$$

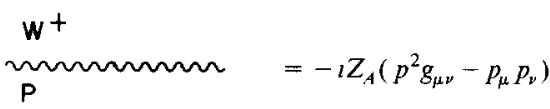

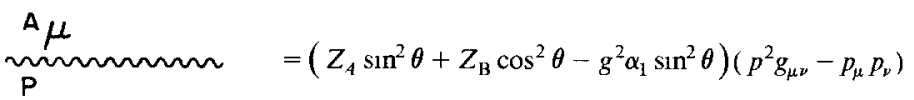

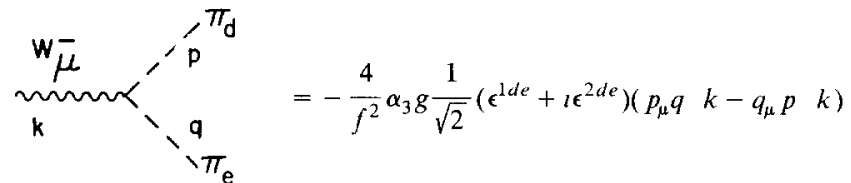

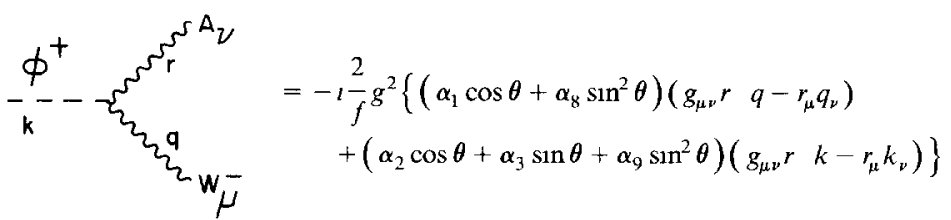

$$
\begin{aligned}
& \pi_{\mathrm{a}} \quad \pi_{\mathrm{b}} \quad=t \frac{1}{f^{4}}\left\{2 \beta_{3}\left(s \delta_{a b} \delta_{c d}+\text { perms }\right)\right. \\
& +\left[\left(\left(8 \alpha_{5}+2 \alpha_{21}+4 \alpha_{20}\right)\left(s^{2}+t^{2}+u^{2}\right)\right.\right. \\
& -\left(8 \alpha_{5}+2 \alpha_{20}-4 \alpha_{4}\right) s(s+t+u) \\
& +\left(4 \alpha_{4}-8 \alpha_{5}-2 \alpha_{21}+4 \alpha_{23}\right)\left(u^{2}+t^{2}\right) \\
& -4 \alpha_{4}\left(p_{1}^{4}+p_{2}^{4}+p_{3}^{4}+p_{4}^{4}\right) \\
& \left.\left.+\left(8 \alpha_{5}-4 \alpha_{4}\right)\left(p_{1}^{2}+p_{2}^{2}\right)\left(p_{3}^{2}+p_{4}^{2}\right)\right) \delta_{a b} \delta_{c d}+\text { perms }\right] \\
& +\sin \theta\left[\delta _ { a b } \delta _ { 3 c } \delta _ { 3 d } \left(-8 \alpha_{6} u t+\left(-4 \alpha_{6}-8 \alpha_{7}-4 \alpha_{19}\right) s(u+t)\right.\right. \\
& \left.\left.\left.+8 \alpha_{19}\left(p_{1}^{2}+p_{2}^{2}\right)\left(p_{3} p_{4}\right)\right)-64 \delta_{3 \alpha} \delta_{3 b} \delta_{3 c} \delta_{3 d} \alpha_{10}(s t+s u+u t)\right]\right\}
\end{aligned}
$$


no particular gauge-fixing term is needed At the two-loop level there are two kinds of heavy Higgs mass effects one is proportional to $M_{\mathrm{H}}^{2}$ and the other is proportional to $\ln \left(M_{\mathrm{H}}\right)$ The $M_{\mathrm{H}}^{2}$ effects come from loop diagrams which contain virtual gauge particles and, when this is the case, one needs to choose a gauge-fixing term and then the background gauge technıque makes the difference

The one-loop computation of the processes needed to determine the remaining coefficients of the above structures has been summarized in table 5 , the corresponding calculations obtained from the structures of the effective lagrangian have been written in table 6 A companson of the results summarized in tables 5 and 6 gives the necessary equations to determine the coefficients of the structures of the effective lagrangian The numencal value of the coefficients is the following

$$
\begin{aligned}
& \beta_{1}=-\frac{1}{(4 \pi)^{2}} \frac{3}{8} \tan ^{2} \theta_{\mathrm{W}} \ln \frac{M_{\mathrm{H}}^{2}}{M_{\mathrm{W}}^{2}}, \quad \beta_{2}=\frac{1}{(4 \pi)^{2}}\left(\frac{M_{\mathrm{H}}^{2}}{2 f^{2}}+\frac{3}{4} g^{2} \ln \frac{M_{\mathrm{H}}^{2}}{M_{\mathrm{W}}^{2}}\right), \\
& \beta_{3}=-\frac{1}{(4 \pi)^{2}} \frac{3}{2} M_{\mathrm{H}}^{2} \ln \frac{M_{\mathrm{H}}^{2}}{M_{\mathrm{W}}^{2}}, \quad \alpha_{1}=-\frac{1}{(4 \pi)^{2}} \frac{1}{12} \tan \theta_{\mathrm{W}} \ln \frac{M_{\mathrm{H}}^{2}}{M_{\mathrm{W}}^{2}}, \\
& \alpha_{2}=-\frac{1}{(4 \pi)^{2}} \frac{1}{24} \tan \theta_{\mathrm{W}} \ln \frac{M_{\mathrm{H}}^{2}}{M_{\mathrm{W}}^{2}}, \quad \alpha_{3}=-\frac{1}{(4 \pi)^{2}} \frac{1}{24} \ln \frac{M_{\mathrm{H}}^{2}}{M_{\mathrm{W}}^{2}}, \\
& \alpha_{4}=\frac{1}{(4 \pi)^{2}} \frac{1}{12} \ln \frac{M_{\mathrm{H}}^{2}}{M_{\mathrm{W}}^{2}}, \quad \alpha_{5}=-\frac{1}{(4 \pi)^{2}} \frac{1}{48} \ln \frac{M_{\mathrm{H}}^{2}}{M_{\mathrm{W}}^{2}}, \\
& Z_{A}=-\frac{1}{(4 \pi)^{2}} \frac{1}{4} \ln \frac{M_{\mathrm{H}}^{2}}{M_{\mathrm{W}}^{2}}, \quad \alpha_{21}=\frac{1}{(4 \pi)^{2}} \frac{3}{4} \ln \frac{M_{\mathrm{H}}^{2}}{M_{\mathrm{W}}^{2}}, \\
& \alpha^{2} \ln \frac{M_{\mathrm{H}}^{2}}{M_{\mathrm{W}}^{2}}, \quad Z_{B}=-\frac{1}{(4 \pi)^{2}} \frac{1}{12} g^{2} \tan ^{2} \theta_{\mathrm{W}} \ln \frac{M_{\mathrm{H}}^{2}}{M_{\mathrm{W}}^{2}}
\end{aligned}
$$

All other coefficients have no large Higgs mass dependence and are therefore neglected The subtraction scale $\mu$ has been set at $\mu^{2}=M_{\mathrm{W}}^{2}$

\section{REMARKS}

The calculation of $\pi_{3} \phi_{+} \mathrm{W}_{-}$is a check of the consistency of the background gauge technique The graphs which contribute to this process contain internal gauge fields and are shown in fig 1 The result of this calculation is

$$
\frac{1}{(4 \pi)^{2}}\left\{\frac{3}{4} g^{3} \tan ^{2} \theta_{\mathrm{W}} \ln \frac{M_{\mathrm{H}}^{2}}{M_{\mathrm{W}}^{2}} p_{\lambda}+\left[\frac{3}{8} g^{3} \ln \frac{M_{\mathrm{H}}^{2}}{M_{\mathrm{W}}^{2}}+\frac{g}{4} \frac{M_{\mathrm{H}}^{2}}{f^{2}}\right](p-k)_{\lambda}\right\}
$$



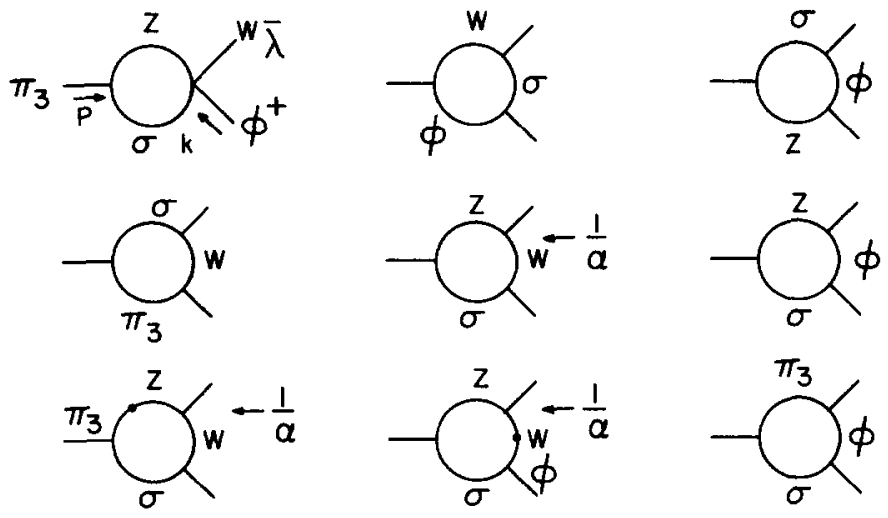

Fig 1 One-boson-loop contributions to the large Higgs mass effects of the $\pi_{3} \phi_{+} W_{-}$process All the factors $1 / \alpha$ have to be cancelled before setting $\alpha$ equal to zero

Using the effective lagrangian, the result would be

$$
-2 g^{3} \beta_{1} p_{\lambda}+\frac{1}{2} g \beta_{2}(p-k)_{\lambda}
$$

where $\beta_{1}$ and $\beta_{2}$ are the coefficients of the only two structures $\mathscr{L}_{b 1}^{\prime}$ and $\mathscr{L}_{b 2}^{\prime}$ which contribute to this process By comparing the two results, the values for $\beta_{1}$ and $\beta_{2}$ can be determined These values are consistent with the previous calculation in which only loops with internal scalar fields are considered

It is important to note that the process $\pi_{3} \phi_{+} W_{-}$can also be calculated in the nonlınear $\boldsymbol{\sigma}$-model, and the result [5] would be

$$
\frac{1}{(4 \pi)^{2}}\left\{\frac{3}{4} g^{3} \tan ^{2} \theta_{\mathrm{W}} \ln \frac{M_{\mathrm{H}}^{2}}{M_{\mathrm{W}}^{2}} p_{\lambda}-\frac{3}{8} g^{3} \tan ^{2} \theta_{\mathrm{W}} \ln \frac{M_{\mathrm{H}}^{2}}{M_{\mathrm{W}}^{2}}(p-k)_{\lambda}\right\},
$$

where $1 / \epsilon$ has been replaced by $\ln \left(M_{\mathrm{H}} / M_{\mathrm{W}}\right)$ One can note that the coefficient of $(p-k)_{\lambda}$ cannot be accounted for by the structure $\mathscr{L}_{2}^{\prime}$, which is the only symmetric structure that contributes to the momentum $(p-k)_{\lambda}$ of the $\pi_{3} \phi_{+} \mathrm{W}_{-}$process Thus, if the nonlinear $\sigma$-model had been used in this calculation, then the coefficient $\beta_{2}$ of $\mathscr{L}_{b 2}^{\prime}$, would be inconsistent

Another example, where a computation in the nonlinear model fails, is the W boson self-energy In the nonlinear model, the one-loop scalar diagram, which contributes to the W self-energy, is that of fig 2 A calculation [2] gives

$$
-\frac{l}{(4 \pi)^{2}} \frac{1}{12} g^{2} \ln \frac{M_{\mathrm{H}}^{2}}{M_{\mathrm{W}}^{2}}\left(p^{2} g_{\mu \nu}-p_{\mu} p_{\nu}\right)
$$

where $1 / \epsilon$ has been replaced by $\ln \left(M_{\mathrm{H}}^{2} / M_{\mathrm{W}}^{2}\right)$ 


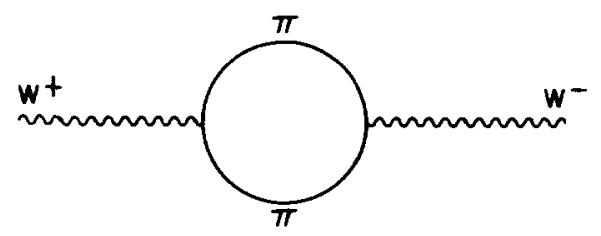

Fig 2 The one-loop scalar contribution to the W self-energy in the nonlınear $a$-model

The scalar diagrams in the linear model, shown in fig 3, contribute to the W self-energy The diagram at the top of fig 3 is the same as that of fig 2, it gives an infinity but it can be renormalızed The bottom diagram gives the true contribution to the heavy Higgs mass effect A calculation gives

$$
+\frac{l}{(4 \pi)^{2}} \frac{1}{12} g^{2} \ln \frac{M_{\mathrm{H}}^{2}}{M_{\mathrm{W}}^{2}}\left(p^{2} g_{\mu \nu}-p_{\mu} p_{\nu}\right)
$$

which is a result different from that of fig 2 In this case the difference is in the sign The discrepancy in the last two calculations can be interpreted as a discrepancy in the correspondence between the infinities of the nonlinear $\sigma$-model and the $\ln \left(M_{\mathbf{H}}\right)$ of the linear model, in the presence of gauge fields
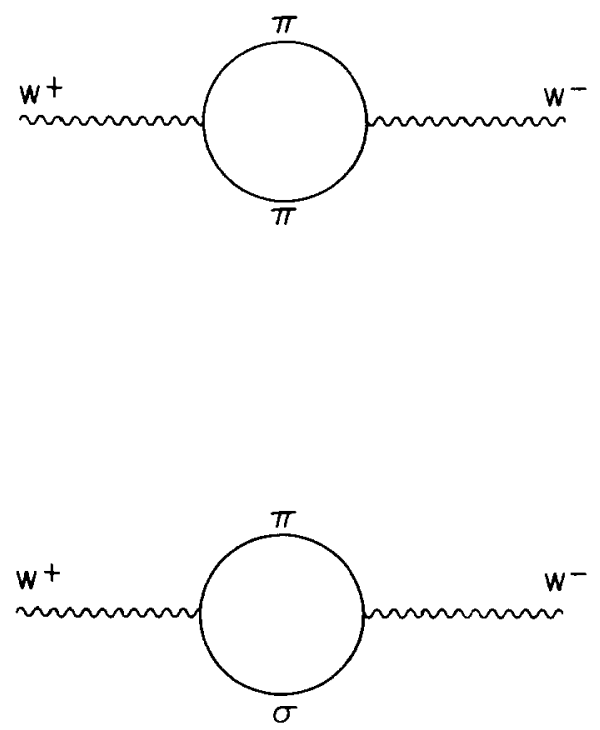

Fig 3 The one-loop scalar contribution to the W self-energy in the linear $\sigma$-model 


\section{Conclusions}

The effective lagrangian contains all possible processes at a given loop order The equations of motion show that the presence of a heavy fermion and a heavy Higgs boson can give rise to invariant constrants for the fields of the lighter particles of the theory If these constraints are put in the linear lagrangian, a nonlinear lagrangian is obtained

At the tree level, the effective lagrangian is the nonlinear lagrangian At one loop and beyond the effective lagrangian can be written as a sum of all possible symmetric structures There is only a finite number of possible structures at a given loop order The symmetries of these structures can be found from an expansion of the lagrangian in the path integral The coefficients of these structures are found by calculatıng a relatively small number of graphs at the desired loop order Once all coefficients are found, the effective lagrangian is fully known and any process can be read from it In order to preserve the explicit gauge invariance of the effective lagrangian, the background gauge field technique [4] was used

Discrepancies arise when the large mass effects of the linear model are compared with the infinities of the nonlinear model at one loop These discrepancies are more evident when gauge fields are present A nonlinear constrant for the fields can be obtained from the linear $\sigma$-model lagrangian by letting the mass $M_{\mathrm{H}}$ of the $\sigma$-particle go to infinity Then in the path-integral the factor $\left(2 \Phi^{\dagger} \Phi-f^{2}\right)$ of the potential

$$
\frac{M_{\mathrm{H}}^{2}}{8 f^{2}}\left(2 \Phi^{\dagger} \Phi-f^{2}\right)^{2}
$$

goes to zero In general, at the limit of infinite $M_{\mathrm{H}}$ any potential of the form

$$
\frac{M_{\mathrm{H}}^{m}}{8 f^{m+4 n-4}}\left(2 \Phi^{\dagger} \Phi-f^{2}\right)^{2 n}
$$

where $m$ and $n$ are positive integers, will result in the constraint $2 \Phi^{\dagger} \Phi-f^{2}=0$ for the $\Phi$ fields When this constraint is put in the lagrangian it gives rise to the nonlınear $\sigma$-model One can note that the same nonlınear $\sigma$-model is obtained for all the possible potentials Thus, the resulting nonlınear $\sigma$-model is the limit for many models, one model for each potential The non-uniqueness of the infinite $M_{\mathrm{H}}$ limit may give rise to the discrepancies between the linear and nonlinear models at finite $M_{\mathrm{H}}$

Out of the many possible models with potentials of the type described above, only the trees of the linear $\sigma$-model may be shown to have a one-to-one correspondence with the trees of the nonlinear $\sigma$-model This is due to the fact that at the limit of large $M_{\mathrm{H}}$ mass, larger than the external momentum $p$, the trees of the linear 
o-model are independent of $M_{\mathrm{H}}$ Thus, the trees of the linear model do not change when $M_{\mathrm{H}}$ is brought to infinity

At the one-loop level and beyond the graphs of the linear $\sigma$-model are dependent on the logarithm or a power of $M_{\mathrm{H}}$, these graphs lose meaning when $M_{\mathrm{H}}$ goes to infinity Also, the graphs of the other models, with non-renormalizable potentials, depend on $M_{\mathrm{H}}$ Therefore, no general correspondence between these models and the nonlınear $\sigma$-model should be found at finite values of $M_{\mathrm{H}}$

In the presence of a heavy fermion, the $\mathrm{SU}(2)_{\mathrm{L}}$ invariant constraints

$$
t_{\mathrm{R}}=0 \quad \text { and } \quad\left(\phi_{0}^{*} \phi_{+}\right)\left(\begin{array}{l}
t \\
b
\end{array}\right)_{\mathrm{L}}=0
$$

arise A nonlinear fermion model can be obtained The lagrangian of this nonlinear model is the effective lagrangian at the tree level The treatment of this case is analogous to the treatment of the heavy Higgs boson and the same conclusions apply

In this paper, the one-loop calculation for external scalar and vector bosons in the presence of a heavy fermion and a heavy boson has been accomplished When only the fermion is considered as heavy the theory is much more complex, the one-loop case of external scalar bosons has been considered by Steger et al The more general case of external fermions, scalar and vector bosons is under consideration

In this paper nonlinear models have been used in the derivation of the effective lagrangian The effective lagrangian contains trees only, thus, nonlinear models can be safely used for this purpose Subsect 32 shows some physical effects for the scalar and vector bosons due to the presence of a heavy fermion This theory considers a fermion family that has one light and one heavy quark, thus it is applicable to the study of a heavy top quark The heavy quark effects are proportional to $M^{2}$ and $\ln \left(M^{2}\right)$ and can be of importance in the search for the heavy top quark

It is a pleasure to acknowledge the guidance and help of York-Peng Yao who also suggested the topic of this research There were useful discussions and checks of the calculation with Herber Steger and Alfred Hill

\section{References}

[1] H Steger, E Flores and York-Peng Yao, Phys Rev Lett 59 (1987) 385

[2] T Appelquist and C Bernard, Phys Rev D22 (1980) 200, D23 (1981) 425

[3] R Akhoury and York-Peng Yao, Phys Rev D25 (1982) 3361

[4] L F Abbott, Nucl Phys B185 (1981) 189

[5] A C Longhitano, Nucl Phys B188 (1981) 118 